\title{
Application of Bayesian methods and networks to ignition hazard event prediction in nuclear waste decommissioning operations
}

\author{
A.F. Averill ${ }^{1}$, J.M. Ingram, P.G. Holborn, P. Battersby and C.M. Benson. \\ Hydrogen Hazards Unit. London South Bank University (LSBU), London SE1 0AA UK.
}

\begin{abstract}
The major purpose of the study is to examine how Bayesian networks can be used to represent and understand potential ignition scenarios in nuclear waste decommissioning. This is illustrated using a network to represent a situation with stacked storage boxes containing pyrophoric material removed from waste storage silos. Corrosion of this material during storage produces hydrogen which is released through a filter medium into the gap between the boxes. The probabilistic relationships used to indicate dependence between network nodes are expressed by conditional probability tables or $\mathrm{C}++$ coded equations that relate to UK nuclear industry corrosion and storage data. The study focuses on optimal prediction of the likelihood of a flammable hydrogen atmosphere arising in the gap between stacked boxes and the conditions necessary to exceed the lower flammable limit. It is concluded that the approach offers a useful means of easily determining the manner in which varying the controlling parameters affects the possibility of an ignition event. The effect of data variation can be examined at first hand using the supplementary Bayesian Network that accompanies the article.
\end{abstract}

Keywords: Nuclear-waste; Magnox-corrosion; Hydrogen-ignition; Bayesian-Networks.

\section{Introduction}

Hydrogen explosion hazards have been a particular concern relating to waste storage decommissioning and reprocessing operations which involve Magnox containing waste [1]. Corrosion of magnesium containing material in the waste sludge, together with the effects of radiolysis, produces hydrogen gas which can be held in pockets enveloped in the sludge. If these pockets are disturbed during processing or storage operations there is the possibility of developing a flammable atmosphere in the ullage space above the sludge. A useful discussion and survey of reactive metal corrosion during nuclear waste packaging has been provided by Serco Technical Consulting Services in an extensive report for the Nuclear Decommissioning Authority [2]. Details of the corrosion and storage of Magnox-containing waste in the UK are also available from a report made available in the public domain by Nuclear Technologies plc [3].

There are a variety of possible ignition sources that can develop during decommissioning, including electrostatic (Ingram et al. [4]) and mechanical (Jones et al. [5], Averill et al. [6-10]). Of particular importance are those relating to surface heating or sparking caused by mechanical stimuli; e.g. sliding contact or impacts involving metal bodies or a metal

\footnotetext{
${ }^{1}$ Corresponding author. E-mail address averilla@1sbu.ac.uk
} 
body with a concrete silo wall. The presence of pyrophoric magnesium-containing material poses a much enhanced risk of an ignition source occurring. Averill et al. [9] have discussed the complex uncertainties involved in determining the ignition probabilities with pyrophoric surface substances present and suggested a mechanism for the ignition of hydrogen in air atmospheres by pyrophoric $\left(\mathrm{Mg} / \mathrm{O}_{2}, \mathrm{Mg} / \mathrm{N}_{2}\right.$ or $\mathrm{Mg} / \mathrm{iron}$ oxides thermite) reaction. It is also possible, at higher impact energy, for ignition of hydrogen in air atmospheres to occur with clean metal on metal impacts [8].

Underpinned by a continuing body of research, the Hydrogen Hazards Unit at LSBU, has collaborated with Sellafield Ltd to produce a comprehensive Technical Guide to hydrogen safety ${ }^{2}$. This provides comprehensive information concerning the general design principles and calculations relating to hydrogen issues that could arise during nuclear decommissioning in the UK. An important component of the Guide is a road map approach to aid process engineers in recognizing the likelihood of an ignition event occurring. Following this work, the major purpose of this paper is to examine how such road maps can be represented by corresponding Bayesian networks to better understand potential incident scenarios. Bayesian statistics also offers a means of including any prior knowledge that is available, particularly the relevant beliefs held by experts in the field. This is sharply different to classical statistical methods such as experimental design $[6,7]$ where prior information is discarded. Data that becomes available is used to continually update a Bayesian model or network which is initially specified by the prior knowledge.

Bayesian networks effectively mesh together Bayesian theorem probability calculations and graphical theory. They facilitate an immediate visualization of all dependent and independent relationships within the model enabling a wider understanding of the process. An important aspect of these networks is that the conditional probabilities used to represent the uncertainty of the true state of a variable can be changed, with the variable being set to a known value when relevant hard evidence is discovered or the uncertainty updated following new but still uncertain evidence. These changes result in an update of the unknown nodes in the network which involves the application of sophisticated algorithms to carry out complex probability calculus. In this manner, the effect of changing conditions can be seen propagating throughout even highly complicated networks. Backwards analysis through the network occurs as well as forward analysis, thus enabling the full effect of available hard evidence to be easily visualised.

This paper focuses on optimal prediction of the likelihood of a flammable gas atmosphere arising in the gap between stacked waste storage boxes which contain pyrophoric material removed during nuclear decommissioning operations. The model is based on realistic data related to the corrosion and storage of Magnox-containing waste in the UK. Although it deals with a specific application, it is envisaged that a similar approach could easily be developed for other storage and possible ignition scenarios. A summary introduction to the relevant aspects of Bayesian inference and networks is given in the next section.

\footnotetext{
${ }^{2}$ I.ChemE Global Award 2015. Highly commended for outstanding achievement in process safety.
} 


\section{Bayesian conditional probability, likelihood and networks}

In our laboratory ignition experiments [5-10], the probability of ignition was simply determined by the number of times it actually occurred divided by the total number of similar tests carried out. This is clearly useful to deal with situations which replicate the experimental conditions but may be of limited use in the real world where there is uncertainty and the possibility of enormous environmental variation. If there are no previous reported instances or experimental test results of ignitions occurring under the same conditions being considered, an inclusive approach can be adopted based on Bayesian or conditional probability: this can formalise a consensus belief under conditions of uncertainty and utilise all of the information available.

Although Bayes' theorem has in recent years been much discussed in the literature, there can be some difficulty in properly understanding the fundamentally important difference between likelihood and conditional probability. Bayes' theorem can be expressed as a relationship between the prior $P(\mu)$ and the likelihood $P(Y \mid \mu)$ (with a data term $P(Y)$ included as a scaling or normalising parameter) to give an updated posterior $P(\mu \mid Y)$.

$$
P(\mu \mid Y)=P(Y \mid \mu) P(\mu) / P(Y)
$$

Here, the prior represents a probability distribution (hypothesis) of the possible mean value for the data being considered which allows for the incorporation of both preexisting knowledge and expert belief. It is updated by the likelihood ratio $P(Y \mid \mu) / P(Y)$ which considers any future evidence and determines the probability of that data occurring for each possible value of $\mu$ within the distribution. Multiplying these probabilities by the prior distribution then allows the posterior to be obtained which represents the altered and updated probability distribution of the mean value. It should be recognised that likelihood values differ from conditional probability, in that they are not constrained to be mutually exclusive and exhaustive with a total probability value of unity: this makes it necessary for likelihood ratios to be used in the updating process rather than individual likelihoods. The application of likelihood ratios can be illustrated using a simple example involving ignition laboratory tests results. Figure 1 shows the likelihoods (determined from a binomial distribution) for a series of 10,20 and 30 tests in which there were 4 and 8 and 12 positive ignition results. The plots are scaled so that the best supported ignition probability of 0.4 for each set of tests corresponds in each case to a likelihood of 1 . Clearly the increase in number of tests has narrowed down the likely range of the ignition probability: the likelihood of the ignition probability being 0.6 as apposed to 0.4 , for example, is seen from the Plots as greatly decreasing with increase in the number of tests involved. This observation is formalised by comparing the likelihoods with that for the most supported hypothesis for ignition probability. The likelihood ratios for 10, 20 and 30 tests; 2.25, 5.1 and 11.4 directly indicate the extent to which the most supported ignition probability (0.4) is more likely than that under consideration (0.6). Finding additional evidence, in this case by increasing the number of tests, and taking account of the likelihoods enables a modified (posterior) distribution of the ignition probability 
hypothesis to be obtained. When experimental evidence or data is not available, expert belief can be taken into account to formulate the prior distribution.

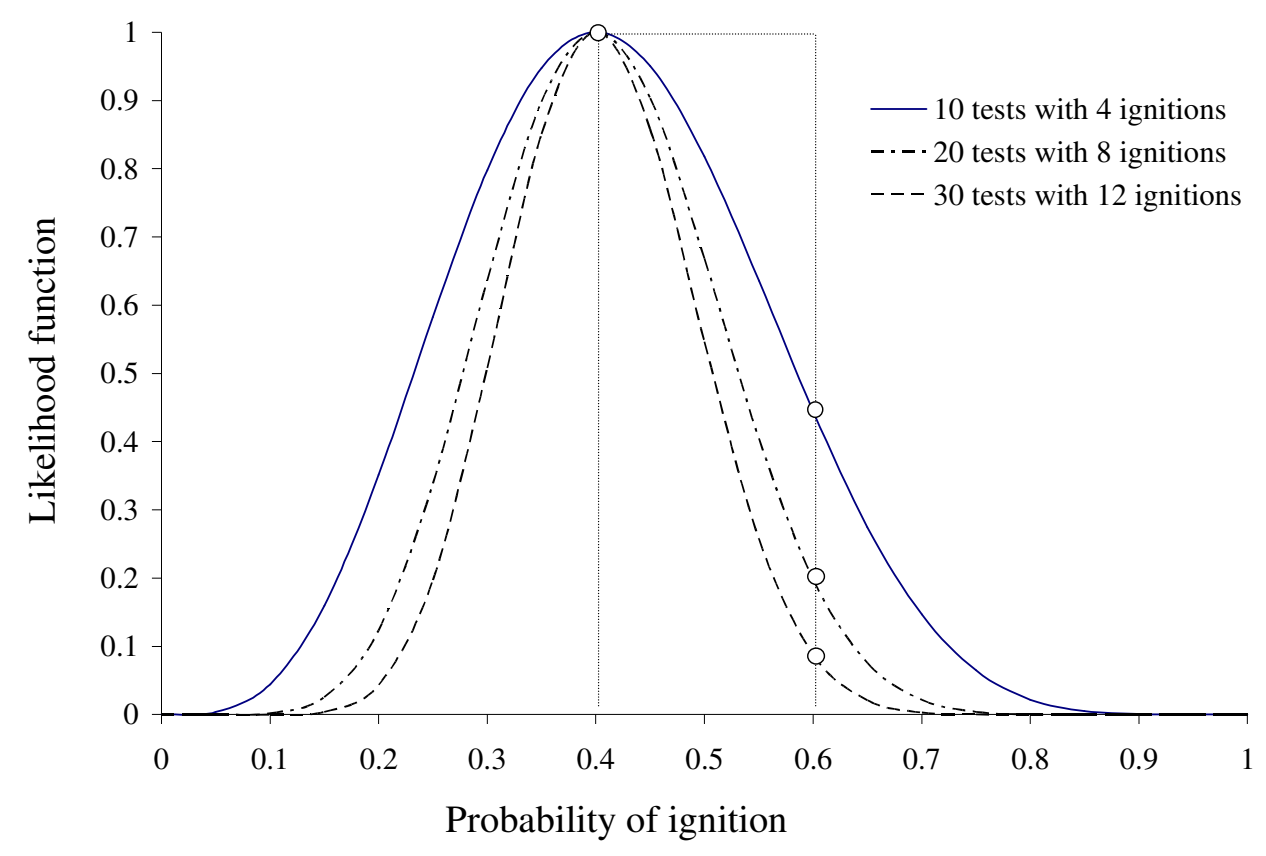

Figure 1. Likelihood function for a series of ignition tests.

\subsection{Belief judgements and expert opinion.}

The philosophical nature of subjective and objective judgement has been much discussed with considerable differences in the conclusions reached. There is indeed little apparent agreement as to what constitutes the difference between objective and subjective judgements or beliefs. For many purposes, expert beliefs can be considered in terms of being epistemically objective in that they relate to the distinction between truth and falsity or accuracy and inaccuracy. Judgments concerning such as degree of beauty, are clearly subjective in that accuracy or truth is not an absolute. There is no measurable scientific way of settling the matter. Although it is a more difficult matter to assess the degree of objectivity in an expert opinion regarding a hypothesis (such as for the most likely probability of an ignition occurring) these judgments will generally be regarded as subjective.

When there is a lack of evidential data on which to base a prior distribution for a parameter, it can be formulated on the basis of subjective expert belief or opinion [1113]. As Kuhnert et.al. [11] point out, the uncertainty expressed by the experts may be epistemic because of lack of knowledge or be due to natural variation: in most cases it is likely to represent a combination of both. If the natural uncertainty outweighs the 
epistemic uncertainty then further research effort to reduce the uncertainty may be futile. The aggregation of opinions from multiple experts can be performed in many different ways allowing the overall uncertainty to be determined and an estimate of a standard error to be made. If the elicitation of one expert alone is sought then the expert, apart from having a high degree of subject expertise, will be required to have sufficient knowledge of probability theory to enable the uncertainty to be properly expressed (i.e. in the form of a probability distribution).

Berger [13] has considered in detail the subjective determination of the prior density and outlined various approaches. These include the histogram approach which involves plotting a histogram of the subjective probability of each interval of the unknown variable. This, however, can be difficult to work with and there are difficulties on account of tails being missing from the prior density. Another approach, that of matching a given functional form entails assuming a given functional form and then choosing the density of this distribution which best matches prior beliefs. This usually involves calculation from estimated prior moments $(\mu, \sigma$ or in the case of beta distributions, $\alpha, \beta)$ but as Berger points out, this can be an uncertain undertaking due to the fact that the tails of a probability density can have a serious effect on the magnitude of its moments. A better method of establishing prior parameters according to Berger, is to take estimates of several fractiles (quantiles) of the prior distribution and then select parameters which lead to a density that matches these quantiles as closely as possible. Because tables of quantiles of standard densities are readily available, it is easier to estimate these subjectively rather than try to estimate the distribution moments. This approach is particularly useful where only very vague prior information is available and it has the advantage of simpler application. Where possible, it is useful to compare the functional form selected with a comparison of the ratios of intuitive likelihoods at various locations within the distribution. It can easily be seen from the example given earlier concerning the likelihood of ignition test results (Fig.1), that knowledge (or belief) of the likelihood ratios will enable a probability distribution to be quantitatively characterised. This also remains the case for unsymmetrical distributions.

\subsection{Networks}

Bayesian networks are probabilistic graphical models, Directed Acyclic Graphs (DAG) in which system variables (nodes) are connected by directional arrows (arcs). They are directional indicating dependence with an arc from node A to node B implying some causality (i.e. A causes B). The distinction between causal relationship between nodes and that corresponding to mere correlation is an important one and not always easy to determine. It has been pointed out that if the relationship between at least three variables is measured, intuition suggests that one of the variables will virtually control the relationship between the others [14]. Networks can initially be set up in a simple fashion and a more complex system built up by the introduction and combination of more nodes with their own relatively simple relationships. 
Because Bayesian networks are acyclic there are no loops between nodes or any feedback cycle possible. Although nodes can be discrete or continuous they must be consistent in that a continuous defined node cannot be parent to a discrete definition child. Continuous defined nodes are, however, often avoided through a simple process of considering the continuous distribution as a sequence of quantiles with finite intervals over the continuous range: this, as explained above, is a useful way of encapsulating expert belief. The probabilistic relationships, indicating dependence between the nodes, is encoded either by conditional probability tables (CPT) or through the use of equations which specify the tables. This encoding describes how each combination of parental node states, in turn, influences each child state. The root nodes are by nature specified only by prior probability distributions since they are not influenced by any other network nodes. Conditional probabilities assigned to each child node state must take into account the influence of every possible combination of parent node states. The can be determined in a variety of ways, utilising the results of experimental tests or case histories, theoretical analysis or eliciting expert opinion. In some networks it is possible to define the CPT by a process of learning from a collection of cases where these are available.

Once set up, a Bayesian network represents the uncertainty of a variables (e.g. presence of a flammable atmosphere) true state in terms of a number of conditional probabilities. If the condition of the true state becomes known through hard evidence (such as readings from gas sensors) in Bayesian network terms the node is described as instantiated. Instantiation results in immediate updating of the assigned beliefs contained in the uncertain nodes in the network. Where the new evidence (of for example the gas sensor readings) is itself uncertain in nature, it can be incorporated as a "likelihood finding: this, like instantiation, results in an update of the unknown nodes in the network. The effect of new evidence propagating throughout the network represents a major advantage in using them to more fully understand the phenomena being modelled. To properly understand how this relates to conditional dependence and independence (d-separation) in the network it is necessary to recognise that parent nodes become marginally independent when the child node is hidden (i.e. unknown) and become dependent when it is known. In the case of a root node, the child nodes are dependent when the root node is hidden and conditionally independent when it is known.

A further important aspect of using Bayesian networks is in decision making (e.g. in the design process of a decommissioning operation) where utility functions are incorporated which express the extent to which desired outcomes are achieved. The information they contain becomes more readily visible to the design engineer rather than being buried amongst detailed data. 


\section{Implementation of the Bayesian network}

There is a wide range of network software available including generalised mathematical tools with network toolboxes (e.g. MatLab ${ }^{3}$ and Mathematica ${ }^{4}$ ) and more specialised software such as Netica ${ }^{5}$ and Hugin ${ }^{6}$ for the creation and analysis of Bayesian networks. The ease of use and highly intuitive graphical user interfaces of these specialised packages make them extremely attractive to the practising process safety engineer or analyst. An illustrative network will be presented here based upon a hypothetical waste storage operation scenario, using Netica software. The network represents the acceptability of storage conditions (in relation to the development of a flammable atmosphere) following the removal of pyrophoric material containing waste sludge from a nuclear waste silo.

In the scenario being considered, 1 metre cubed stacked boxes are being used for the long term storage of nuclear waste material which contains Magnox. This is continuing to corrode within the boxes generating hydrogen gas as bi-product. The gas rises through a covering water layer into the $100 \mathrm{~mm}$ depth ullage space and is released through a filter: the buoyant gas then flows through horizontal gaps between the stacked boxes creating the possibility of an ignition hazard. As well as controlling the possibility of hydrogen gas concentration increasing sufficiently to form a flammable mixture either in the box itself or in the gap between boxes, there are other major concerns outside the scope of this article: maintenance of the water content of the boxes and prevention of radionuclide escape.

A schematic view of the arrangement considered is shown in Figure 2 where an upper box is seen positioned above a lower chamber to create a gap. Using a simple experimental arrangement with a sub-micron stainless steel mesh filter medium and containing the stacked boxes within a larger enclosure to minimise convection effects, initial experiments to measure gas concentration distribution between the chambers indicated a number of influences controlling the gas dispersion. The indicated effects of gas generation flow rate, gap height between stacked boxes and the size and position of the filter were first encapsulated into the generalised Bayesian network shown in Figure 3 .

\footnotetext{
${ }^{3}$ MathWorks and Toolbox from MIT Strategic Eng.

${ }^{4}$ Wolfram Research. Inc

${ }^{5}$ Norsys Software Corp

${ }^{6}$ Hugin Expert A/S
} 


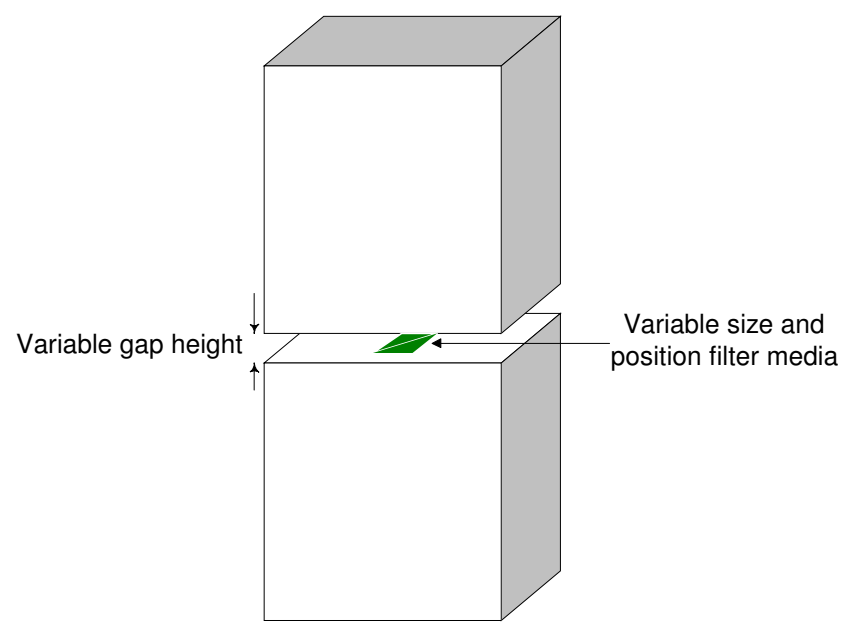

Figure 2. Schema of the waste storage stacking

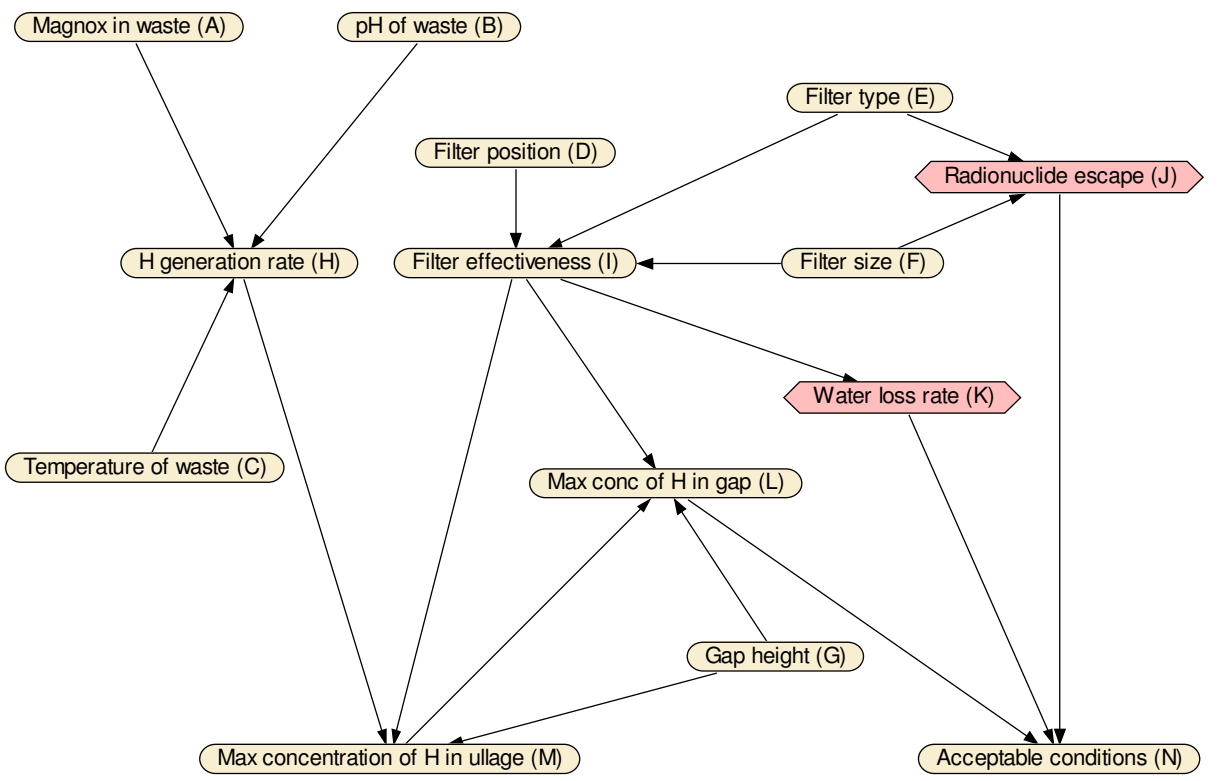

Figure 3. A Bayesian network for storage of nuclear waste in stacked waste boxes.

This involved the combination of expert opinion summarising the known data and underlying science. On scientific grounds, it is very clear as to what parameters control the Magnox corrosion rate: i.e. $\mathrm{pH}$, temperature and amount of exposed material. The other relationships took into account unreported (at this stage) experimental results at LSBU and elsewhere as well as scientific principles. A network like this can be tested for validity and consistency by the later introduction of evidence or if available case study.

As can be seen, there are seven root notes $(A-G)$ for which prior information must be specified. Nodes A, B and C the Magnox ( $\mathrm{Al}$ 80) content of the waste, $\mathrm{pH}$ and temperature determine the hydrogen generation rate in the lower child box $(\mathrm{H})$. This, 
together with the filter effectiveness and gap height, governs the maximum concentration of hydrogen in the ullage (M) and gap space (L). The state of the Acceptable conditions node $(\mathrm{N})$ depends upon the levels of radionuclide escape $(\mathrm{J})$ and water loss rate $(\mathrm{K})$ as well as the hydrogen concentration in the gap being less than the lower flammable limit.

The cathodic reaction which occurs during the Magnox corrosion process produces both hydrogen gas and hydroxyl ions according to:

$$
2 \mathrm{H}_{2} \mathrm{O}+2 \mathrm{e}^{-} \rightarrow \mathrm{H}_{2}+\mathrm{OH}^{-}
$$

concomitant with the anodic dissolution reaction:

$$
\mathrm{Mg} \rightarrow \mathrm{Mg}^{2+}+2 \mathrm{e}^{-}
$$

In neutral unbuffered solutions, such as would initially be the case with Magnox waste dumped into silos and covered with water, the $\mathrm{pH}$ in the vicinity of the metal surfaces will be shifted in the alkaline direction as corrosion progresses until it reaches 10.4 corresponding to a saturated $\mathrm{Mg}(\mathrm{OH})_{2}$ solution. A partially protective hydroxide surface film (small random orientated crystals of brucite [2]) forms at $\mathrm{pH}$ values exceeding 11 which could result from dosing with $\mathrm{NaOH}$ :

$$
\mathrm{Mg}^{2+}+2 \mathrm{OH}^{-} \rightarrow \mathrm{Mg}(\mathrm{OH})_{2}
$$

This will reduce the corrosion and hydrogen generation rate, behaviour that can be incorporated into the network by introducing probabilistic equations with conditional IF THEN ELSE relationships dependent on the expected $\mathrm{pH}$ value (including the effect of dosing with $\mathrm{NaOH}$ ).

An important issue is that small amounts of anions or heavy metal cations can have a major effect on the corrosion rate making it difficult in the absence of direct data to predict the hydrogen generation rate during storage. The amount of uncorroded Magnox contained in the waste is also not likely to be known with any certainty but can be assessed on the basis of known information relating to the contents and conditions of the particular decommissioned waste storage silo and the storage time as well as any observations made. It is the surface area of the metal per unit volume of waste that determines the amount of Magnox available for reaction to generate hydrogen. In consequence, it is a major factor in determining the hydrogen generation rate from a given volume of waste. Magnox corrosion in nuclear waste appears to change when the temperature reaches and exceeds a temperature of somewhere between 55 to $65^{\circ} \mathrm{C}$. At lower temperatures there seems to be relatively little dependence on temperature whereas at higher temperatures there is a strong dependence. Much more difficult to take into account is "Breakaway" which could occur when waste is being disturbed during its transfer from silos into boxes. Breakaway is a term used to denote an increase in corrosion rate brought about by fracture of the protective layer where there is an inability for the surface to reform a protective film. If breakaway occurs to any significant extent due to mechanical damage (may also arise if the temperature increases to $80^{\circ} \mathrm{C}$ ), the 
stable long term corrosion and hydrogen generation rates can accelerate and for a time be greatly exceeded.

The Simplified Model of Gas Generation (SMOGG [15]), a tool developed for modelling gas generation from radioactive wastes in the UK National Inventory can be used to determine the hydrogen gas generation rates for specific storage and composition conditions. However, for the purposes of this article the effects of breakaway and temperature on the hydrogen generation rate are generically represented and modelled using appropriate equations between the corresponding parents and child. The waste $\mathrm{pH}$ is taken as the long term value between 11 and 13 which results from dosing with $\mathrm{NaOH}$ in the silos. It follows that long term corrosion rates are appropriate except when breakaway occurs.

A simple model is used here to represent distribution of the amount of uncorroded Magnox in the waste boxes although in practice it might be necessary to replace it with a better fitting multi modal probability distribution. The prior parameters for the model can be obtained, as outlined earlier, by a process of making estimates of several quantiles and then selecting parameters which produces a probability distribution that matches the quantiles as closely as possible. Three beta distributions are shown in Figure 4 representing different possible beliefs and uncertainty relating to the amount of uncorroded Magnox swarf contained in a box of waste. The situation modelled in this paper is for waste collection from a succession of silos that result in a distribution skewed in the manor shown with $\alpha=2$ and $\beta=5$ Here it can be seen that there are more boxes with smaller amounts of Magnox swarf than with large amounts: the mean is greater than the median and mode values due to the uneven distribution of the amounts (and condition) of waste metal in the storage silos being emptied. The two parameters $\alpha$ and $\beta$ which characterise the distributions are related to the mean or expected value $(\mu)$, variance $\left(\sigma^{2}\right)$ and mode $(m)$.

$$
\begin{gathered}
\mu=\frac{\alpha}{\alpha+\beta} \\
\sigma^{2}=\frac{\alpha \beta}{(\alpha+\beta)^{2}(\alpha+\beta+1)} \\
m=\frac{\alpha-1}{\alpha+\beta-2}
\end{gathered}
$$

When parameters $\alpha$ and $\beta$ are equal, the distribution becomes symmetrical, resembling the bell shaped normal distribution: with skewed distributions as required here, the values of $\alpha$ and $\beta$ will always differ. Whatever the value of the parameters, the continuous beta function always takes a value between 0 and 1 which is used to model the belief probability. It is often the case that is easier and more convenient to specify the mean and variance first and then translate them into the corresponding beta distribution parameters. 


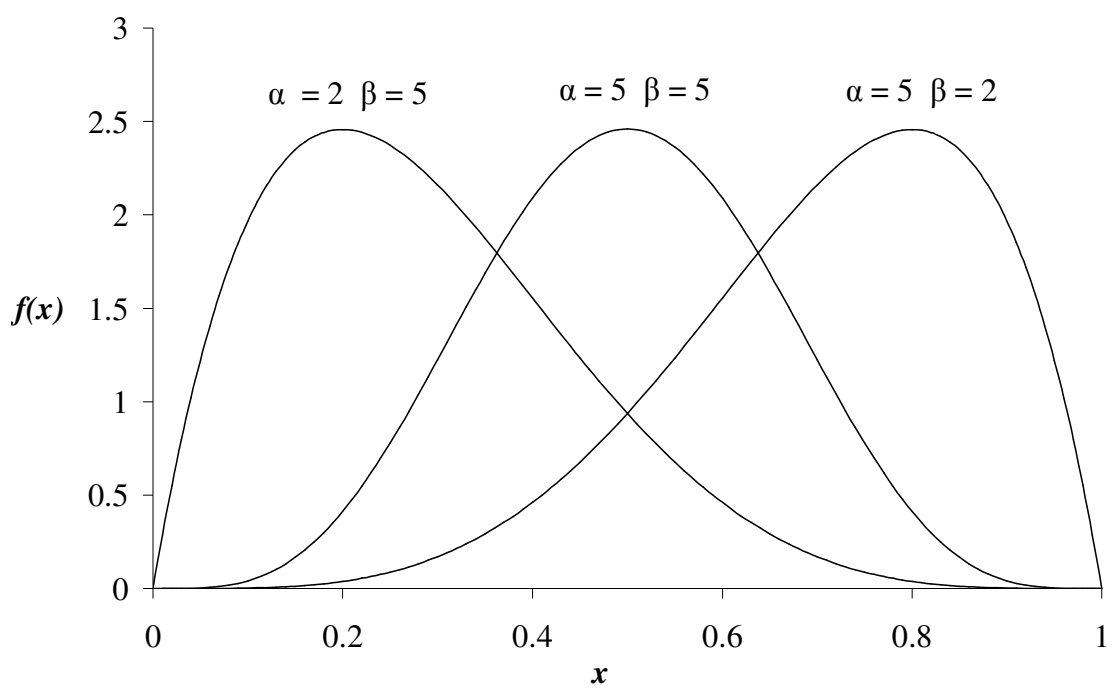

Figure 4. Beta probability distributions representing belief in the distribution of uncorroded Magnox in the storage boxes. The variable $x$ is an arbitrarily scaled magnitude of the amount of Magnox.

Conditional probability relationships between children and parent nodes can be directly formulated as a Table or be expressed by deterministic or probabilistic equations. Whatever the form of the equation, the only nodes which can be referenced in the equation are (i) the node which the equation defines, (ii) the parents of this node and (iii) any constant node which is involved. In all cases, the equations must be converted to Tables (CPT) before the network is compiled. Sometimes it is helpful and convenient to define intermediate variables, implementing them as new nodes in order to simplify the equations. Examples of the different ways of expressing node conditionality are included in the NETICA network file for hydrogen concentration development in the gap space ${ }^{7}$ which is supplementary to this article. Although illustrative in nature, the $\mathrm{C}++$ coded equations (available for inspection in the network) reflect real corrosion data [2] and are representational of those which are appropriate for use in the process of the kind described here. It should be noted that in some cases when using the network, due to the variability of the size of the interval bands chosen for the data, the shape of the probability distribution shown will appear as a visual distortion of the real probability density.

The network for the hydrogen concentration in the gap space, shown in Figure 5, consists of 5 parent and 5 child nodes. All of these, except "Gap" (which is a discrete variable), represent continuous variables and show software generated \% probability values for the states based on the assigned distributions: the figures shown at the bottom of the boxes are the mean and a measure of uncertainty. Figure 5 shows network values before the initial values of the five parent nodes; "Breakaway-mechanical damage to swarf", "Waste temperature (Celsius)," "Area-weight ratio of swarf", "Wt (kg) uncorroded Magnox in box" and "Gap" are updated from later evidence. If, as an example, updating of

\footnotetext{
${ }^{7}$ A limited free version of Netica software (vs. 6.04) able to read and use this network is currently available from Norsys Software Corp.
} 
evidential data indicates that these parent nodes are instantiated to $S$ mall, $45^{\circ} \mathrm{C}, 3,11 \mathrm{~kg}$ and $30 \mathrm{~mm}$ respectively, then the network is changed to that shown in Figure 6 indicating a relatively small chance of a possible flammable atmosphere $(>4 \% \mathrm{H})$ forming within the gap. The maximum hydrogen concentration in the gap space indicates that the probability of forming an atmosphere which just exceeds the lower flammability limit is approximately 0.2 . If, as a further example, sensor evidence is gathered on the $\%$ hydrogen concentration in the ullage space but it is of an unsure nature, it can be introduced as a likelihood finding. This consists of entering a probability for each state of the node which is the probability that the evidential observation would be made if the node were in that state. Entering a probability of 1 indicates that the evidence supports the existing state probability whereas a value of 0 indicates that the state is now considered impossible: intermediate values, of course, represent a supported degree of change. For a gap height of $15 \mathrm{~mm}$, entering the likelihood finding; $0,0,1,1,1 \ldots$, into the node for \%concentration of $\mathrm{H}$ in the ullage space changes the network to that shown in Figure 7. The effect on the probability states for the hydrogen concentration within the ullage can be seen as well as the way that the network has changed other node values to be consistent with the new evidence.

The network described has been shown to offer the means of readily understanding and predicting the effect of future observations on the probability that a flammable atmosphere would arise between the stacked boxes: this with little doubt, is achieved in a much easier manner than could be expected by adopting other approaches.

\subsection{Conclusions.}

A Bayesian network has been described to represent and understand a potential hazard scenario in nuclear waste decommissioning. This relates to an illustrative example of stacked storage boxes (containing pyrophoric material which has been removed from waste storage silos) in which hydrogen is generated through corrosion. The hydrogen generation rate is determined by the amount and condition of the waste material in the boxes and the temperature since the $\mathrm{pH}$ is fixed by previous dosing procedures. Employing realistic values for these parent nodes and taking into account the gap height between the boxes, it is indicated that the lower flammability limit of hydrogen in air $(4 \%)$ is not expected to be exceeded under the most likely storage conditions. 


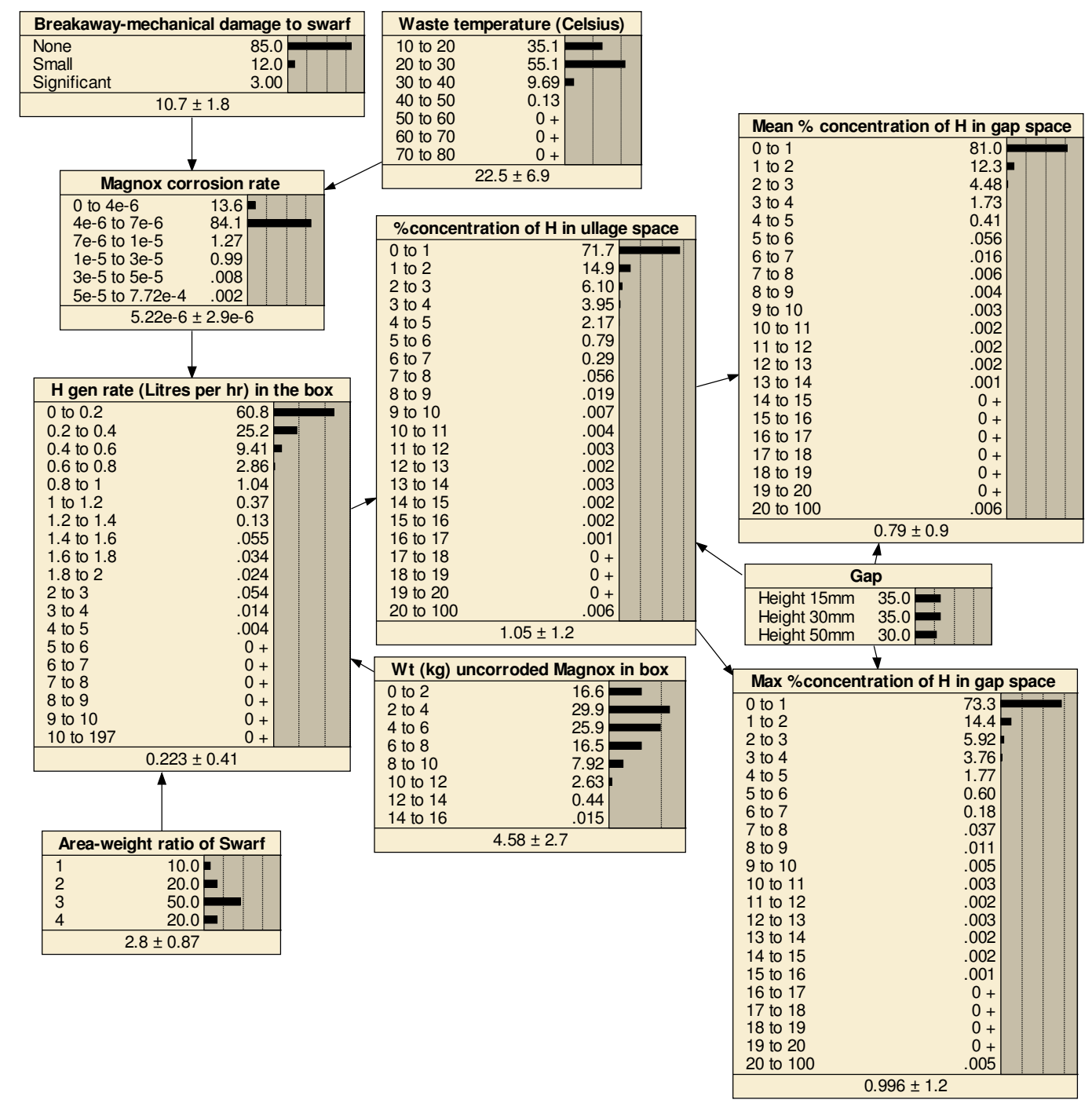

Figure 5. Illustrative Bayesian network with initial values. 


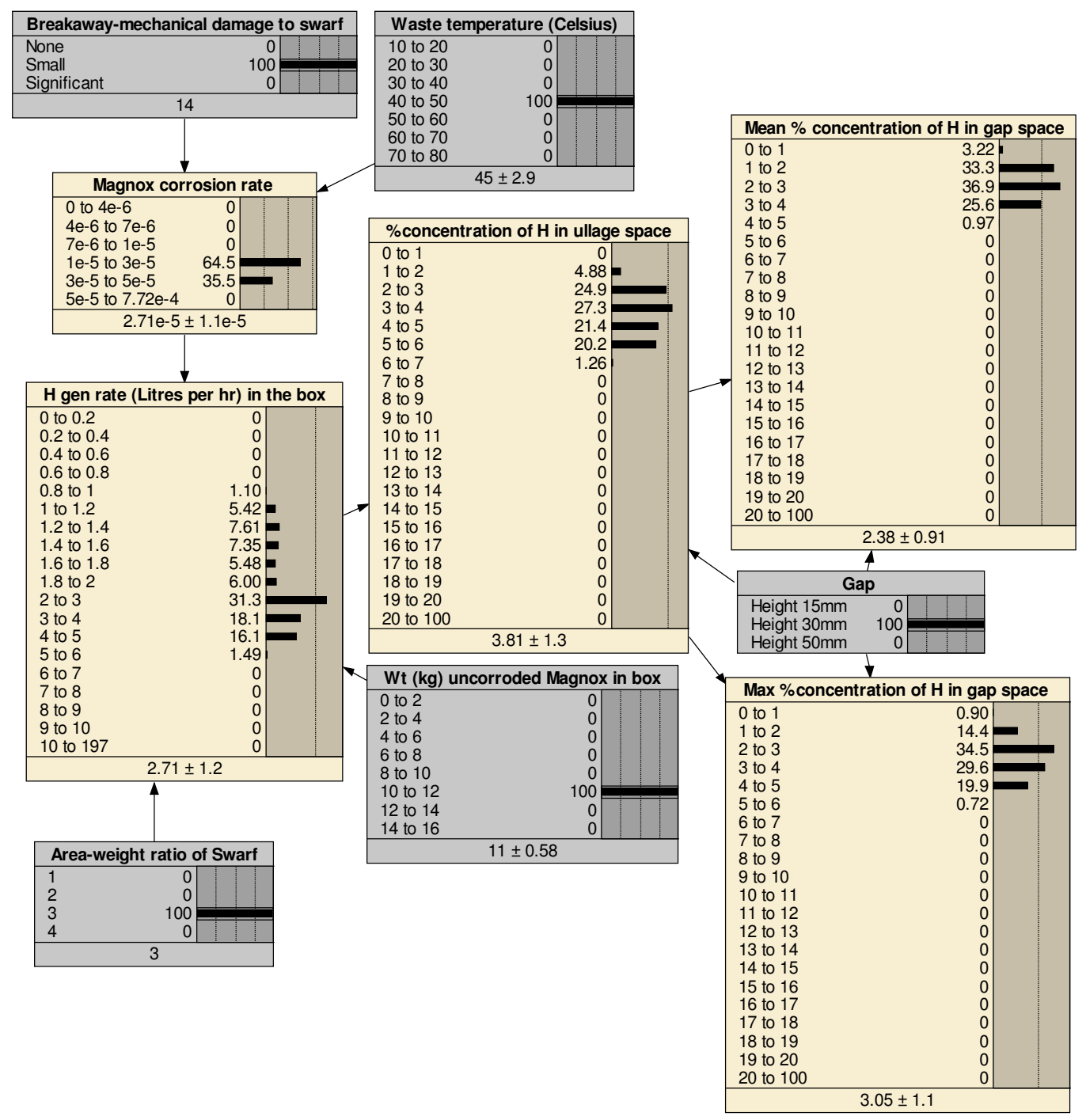

Figure 6. The Bayesian network with instantiated parent values. 


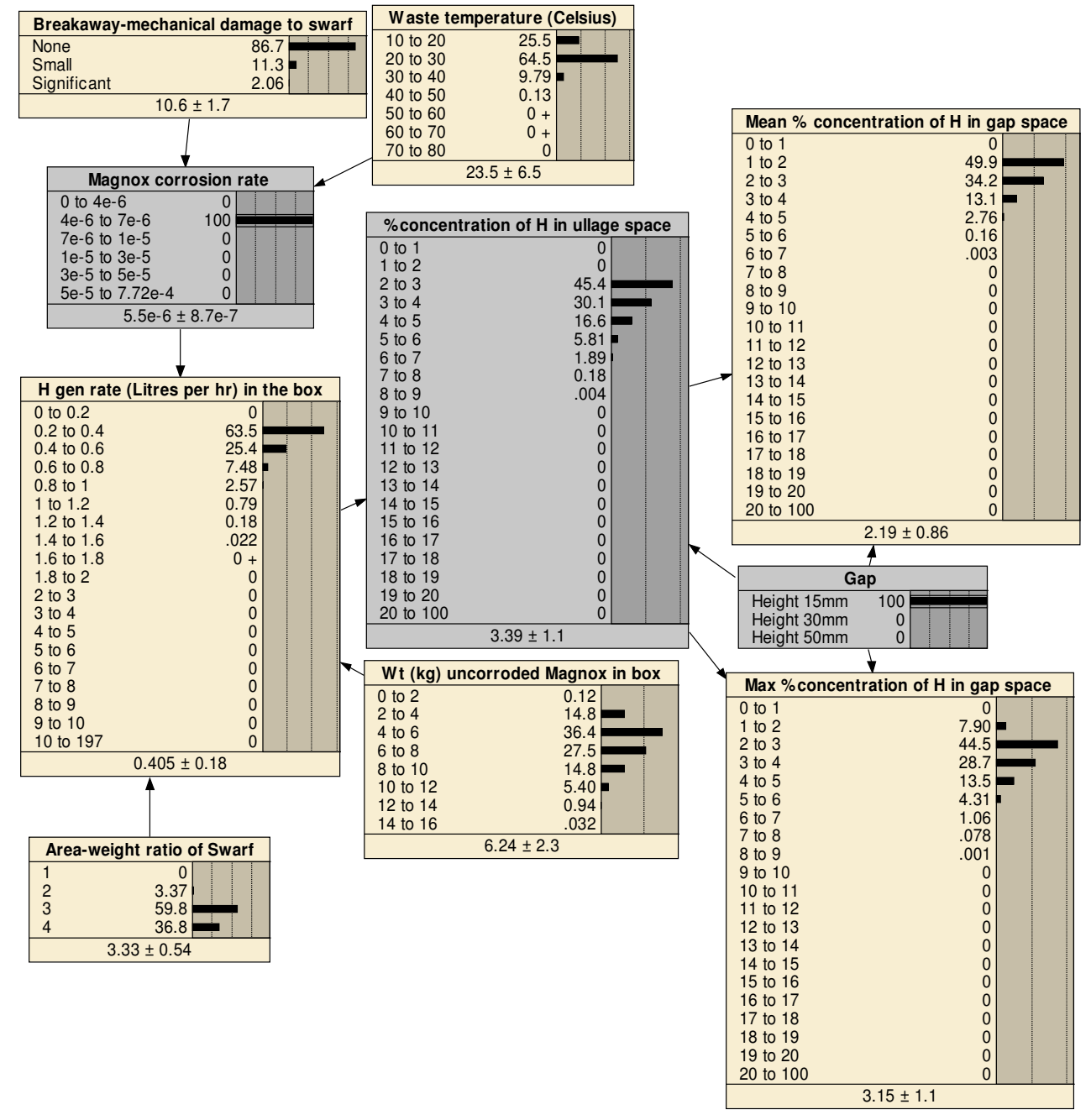

Figure 7. The Bayesian network for a gap height of $15 \mathrm{~mm}$ and likelihood findings entered into the node: \% concentration of $\mathrm{H}$ in ullage space.

\section{Acknowledgments}

The Hydrogen Hazards Unit at London South Bank University would like to acknowledge the financial support of Sellafield Ltd. and the University in undertaking this programme of work. 


\section{References}

1. Kemsell ID, Wakem MJ and Ingram JM. Hydrogen explosions - An example of hazard avoidence and control, In: Hazards XVI. Analysing the past, planning the future. Symposium Series No 148; IChemE; 2001. pp 523-539.

2. A survey of reactive metal corrosion data for use in the SMOGG gas generation model. Report to NDA RWMD. SA/ENV-0895 Issue 2. Sept. 2010. Access online July 2017. https://www.google.co.uk/url? sa=t\&rct=j\&q=\&esrc=s\&source=web\&cd=1\&ved=0ahUK EwjNhNOdhPfUAhUGD8AKHbRWBC0QFggpMAA\&url=https\%3A\%2F\%2Frwm.nda .gov.uk\%2Fpublication\%2Fa-survey-of-reactive-metal-corrosion-data-for-use-in-thesmogg-gas-generation-model\%2F\%3Fdownload\&usg=AFQjCNE9vImf_iZtlJfSswn6fc89EeClg\&cad=rja.

3. The development of a longevity case for packages containing Magnox swarf and fuel element debris. Nuclear Technologies plc. Harwell UK. NT/P386/R156/Final Report 29 April 2006. 16pp.

4. Ingram JM, Averill AF, Battersby P, Holborn PG and Nolan PF. Electrostatic ignition of sensitive flammable materials. Process Safety and Environmental protection: is charge generation due to bubble bursting in aqueous solutions a credible hazard? 2014;92:750759

5. Jones SJ, Averill AF, Ingram JM, Holborn PG, Battersby P, Nolan PF, et al. Impact ignition of hydrogen - air mixtures, In: Hazards XIX. Process Safety and Environmental Protection. Symposium Series No 151; IChemE; 2006. pp 401-409.

6. Averill AF, Ingram JM, Holborn PG, Battersby P and Nolan PF. Ignition of flammable hydrogen/air mixtures by controlled glancing impacts in nuclear decommissioning. Paper No 234, $5^{\text {th }}$ Int.Conf. on Hydrogen Safety, Sept. 9 - 11, 2013, Brussels, Belgium.

7. Averill AF, Ingram JM, Holborn PG, Battersby P and Nolan PF. Ignition of hydrogen/air mixtures by glancing mechanical impact. International Journal of Hydrogen Energy 2014;39:20404-20410.

8. Averill AF, Ingram JM, Battersby P and Holborn PG. Ignition of flammable hydrogen/air mixtures by mechanical stimuli. Part 1: ignition with clean metal surfaces sliding under high load conditions. International Journal of Hydrogen Energy 2014; 39:18472-18479.

9. Averill AF, Ingram JM, Battersby P and Holborn PG. Ignition of flammable hydrogen/air mixtures by mechanical stimuli. Part 2: Ignition under conditions of rust and surface pyrophoric material contamination. International Journal of Hydrogen Energy 2015; 40:4392-4400. 
10. Averill AF, Ingram JM, Battersby $P$ and Holborn PG. Ignition of flammable hydrogen in air (and other $\mathrm{H}_{2} / \mathrm{N}_{2} / \mathrm{O}_{2}$ mixtures) by mechanical stimuli. Part 3: Ignition under conditions of low sliding velocity $(<0.8 \mathrm{~m} / \mathrm{s})$. International Journal of Hydrogen Energy 2015;40:9847-9853.

11. Kuhnert PM, Martin TG and Griffiths SP. A guide to eliciting and using expert knowledge in Bayesian ecological models. Ecology Letters. 2010;13:900-914.

12. Jacobs RA. Methods for combining experts' probability assessments. Neural computation 1995;7:867-888.

13. Berger JO. Statistical Decision Theory and Bayesian Analysis. 2nd Ed. Chapter 3. Springer-Verlag. NY, Berlin, Heidelberg and Tokyo. 1985.

14. Murphy K. A brief introduction to graphical models and Bayesian networks. 1998. http://www.cs.ubc.ca/ murphyk/Bayes/bnintro.html. Accessed 17th October 2016.

15. User guide for SMOGG Version 7.0: A simplified model of gas generation from radioactive wastes. AMEC Foster Wheeler: RWM007. January 2016. 